\title{
ECOEFICIENCIA EN LOS PROCEDIMIENTOS DE LICITACIÓN PARA LA ADQUISICIÓN DE INSUMOS DE USO COTIDIANO POR LA ADMINISTRACIÓN PÚBLICA FEDERAL DE BRASIL
}

\author{
ECO-EFFICIENCY APPLIED TO THE ACQUISITION OF EVERYDAY \\ UTENSILS IN THE BRAZILIAN FEDERAL ADMINISTRATION
}

\begin{abstract}
Maraluce Maria Custódio
Mestre em Direito Constitucional pela UFMG. Mestre em Direito Ambiental pela Universidad Internacional de Andalucía (Espanha). Doutora em Geografia pela UFMG em cotutela com a Université D’Avignon (França). Professora da Graduação e Professora Permanente Do Programa De Pós-Graduação Em Direito Da Escola Superior Dom Helder Câmara - Mestrado Em Direito Ambiental E Desenvolvimento Sustentável. E-mail: malucemc@gmail.com

Márcio Luís de Oliveira

Doutor e Mestre em Direito; Professor Adjunto da Escola Superior Dom Helder Câmara (Bacharelado e Mestrado). E-mail: marcio.luis@uol.com.br
\end{abstract}

Recebido em: 22/06/2015

Aprovado em: 11/04/2016

Doi: $10.5585 / \mathrm{rdb} . v 13 \mathrm{i} 6.272$

\begin{abstract}
RESUMEN: La sostenibilidad ambiental en la vida cotidiana de la Administración Pública debe ser objeto de políticas públicas eficaces, especialmente en la gestión de los bienes públicos. En este sentido, ya través de la metodología deductiva, el paper se centra en el análisis de lasoperacionesde compra de material consumible y gestión de la administración pública cumpliendo los principios del Derecho Ambiental, especialmente del desarrollo sostenible, en conformidad con la Ley de Licitación y la eficiencia ambiental, por lo que la llamada "licitación sostenible". Es importante señalar, que en este trabajo, se dará énfasis a la Unión, ya que la legislación federal, se presenta más detallada y sistematizada. Por lo tanto, se busca discutir los pasos de las políticas públicas y los principios rectores de la administración pública que conducen una actuación ecoeficiente en sus compras de artículos de uso diario. En conclusión, el paperdemuestra la incapacidad actual de la Administración Pública Federal, en las etapas de la política pública de cumplir losprincipiosnecesarios para su desempeño en esta tarea.
\end{abstract}

Palabras Clave: Administración pública; Principio de la eficiencia; Licitación; sostenibilidad; Ecoeficiencia

ABSTRACT: Environmental sustainability in the State's daily self-management should be the subject of efficient public policies, especially in the management of public assets. In this sense, and through the deductive methodology, the article focuses on the analysis of the operation and management of public administration in dealing with the purchase of everyday equipment, in order to watch out the principles of environmental law, especially the 
sustainable development, in accordance with the bidding laws and eco-efficiency, performing the called "sustainable bidding". It is important to emphasize that, in this work, accent will be given to the Union, because, this federal entity presents more detailed and organized legal system Thus, the article discusses the steps of public policy and the guiding principles of public administration to pursue eco-efficient acts in its purchases of everyday items. In conclusion, the article attests the failure by the Brazilian federal government to observe the stages of public policy and the bidding legal principles in this matter.

Keywords: Public Administration; Principle Of Efficiency; Public Bid; Sustainability; EcoEfficiency.

\section{INTRODUCCIÓN}

El tema de la sostenibilidad ambiental,en la gestión de los intereses públicos y privados, ha recibido una atención progresiva del gobierno, del sector privado y de la sociedad civil. Pero, el Estado es la entidad que, principalmente, lleva a cabo la representación de la soberanía popular. Los procesos y procedimientos relacionados con su autogestión son objeto de estudios sobre el cumplimiento real de las prácticas administrativas sostenibles.

En este sentido, el artículo cuestiona la sostenibilidad ambiental como parámetro de eficiencia en el desarrollo de políticas públicas para la adquisición de materiales fungibles de utilización diaria en la administración pública.

Normalmente, la "licitación sostenible" aparece en la doctrina y la jurisprudencia cuando se trata de grandes inversiones estatales, como en proyectos de infraestructura. Sin embargo, la actividad administrativa en su cotidiano, consume una gran cantidad de productos que individualmente no causan,en niveles impactantes, daños al medio ambiente; Entretanto, a grandes escalas, estas herramientas tienen un potencial perjudicial para el medio ambiente tremendo, si administración y uso no se lo hacen por criterios sostenibles, desde la compra. Ejemplos de estos materiales son losbolígrafos, las gomas, el papel, las lámparas, etc.

Así,el trabajo tiene como objetivo general analizar la elaboración de políticas públicas de organización administrativa estatal, teniendo por baseel principio de eficiencia, en relación a compra de estos productos. En concreto, serán aportados algunos criterios legales y técnicos que permitanlograr resultados de adquisición más sostenibles.

El marco teórico de este trabajo es el empleo delos principios jurídicos de la eficacia y eficiencia ambientalen la administración pública, que deberían regir la realización de la "licitación sostenible", para adquisición de materiales.

De esta manera, el trabajo presentará algunos principios jurídicos y etapas del ciclo administrativo para proceder el análisis de la inserción del principio de la sostenibilidad en la gestión pública. Así, demostrando la posibilidad de hacer adquisición de materiales de usocotidiano por la administración pública garantizando la sostenibilidad. Se señala, que en este paper, sehablaran de las políticas administrativas la Unión, pues ella que presenta una legislación más pormenorizada y regulada del tema.

Se utilizó los métodosinductivo y deductivo, centrándose en el análisis del tema licitación y sostenibilidad en la literatura jurídica, legislación y jurisprudencia brasileños.

Para realizar este análisis, se iniciará una breve descripción del funcionamiento de la gestión en la administración pública, para realizar ulteriormente un breve análisis de los 
principios de la legislación ambiental aplicable al temay por fin, comprender, finalmente, cómo el Gobierno Federal de Brasil abordar la cuestión de la eficiencia ambiental.

En su conclusión, el texto aportarála opinión de los autores si es que hay, o no, por la Administración Pública Federal (Unión), el eficaz cumplimiento de las etapas de ejecución de políticas públicas, con aplicación real de la eficiencia ecológica y otros principios administrativos rectores de su actuación, en las compras de utensilios de uso cotidiano.Concluyendo que ciertamente existe un incentivo legal para aplicación de la ecoeficiencia y la realización del desarrollo sostenible en las licitaciones públicas en Brasil, pero poco se ha hecho, sea por desconocimiento de la norma, sea por falta de penalización por no usarla.

\section{ACTIVIDAD POLÍTICA PARA LA AUTOGESTIÓN DEL ESTADO}

La política, conceptualmente, es un conjunto de acciones, teleológica e ideológicamente motivados por intereses - convergentes o divergentes; públicos o privados bajo la dirección - institucional o no - que trabaja en la toma y aplicación de las medidas con potencial efectividad y repercusión local, regional, nacional o internacional. (OLIVEIRA, 2014)

Así que el punto central de la actividad política es la decisión acerca de cualquier cuestión que pueda tener una repercusión pública. Por lo tanto, que se debate, en la Filosofía y la Teoría Política, puntos como motivos y propósitos de la política, la racionalidad y las técnicas de toma de decisiones políticas, los sistemas y las ideologías políticas, la institucionalización de la actividad política, los mecanismos de control delaactuacióny la legitimidad del poder político.

Sin embargo, en la actualidad, en los sistemas democráticos, los científicos y funcionarios públicos tienen puesto de relieve el tema de la eficiencia en la toma y ejecución de las decisiones políticas. La mayoría de los análisis se han centrado en la calidad de la formulación y aplicación de políticas públicas en las relaciones entre el Estado, la sociedad civil y el sector privado. Por lo tanto, la eficiencia se ha convertido en un parámetro importante para evaluar el interés público(o privado) y su satisfacción, que es considerado importante para la viabilidad de los servicios y las ofertas de bienespara la mejora de la vida pública en las sociedades complejas, dinámicas y plurales del mundo contemporáneo. (OLIVEIRA, 2013)

Sin embargo, el tema de la eficiencia, aún, no ha alcanzado la debida importancia en el desarrollo de políticas públicas cuando se habla de las actividades de auto administracióndel Estado. En este sentido, los órganos estatales han sido ineficientes en el manejo de los recursos y bienes del Estado, substancialmente en las cuestiones que en apariencia son de poca importancia o pequeños valores. Y, se ignora, en especial, el principio de la eficiencia ambiental, punto básico hoy en políticas de Estados que buscan cumplir los requisitos ambientales, con destaque para el desarrollo sostenible, en la gestión de recursos.

La protección del medio ambiente es hoy uno de los puntos más importantes en discusión internacional y nacional. Esencialmente en cuestiones como la falta de agua y el cambio climático. La necesidad de proteger y recuperar el medio ambiente es tema centralen la agenda de los programas gubernamentales y políticos en todos los países, entre ellos, Brasil. 
Los Estados tienen un papel esencial en esta cuestión; principalmente después,dela Declaración de Estocolmo de 1972. Esta obliga los Estados a crearórganos y leyes para controlar el uso del medio ambiente y protegerlo para garantizar la vida en todas sus formas. Como señala Holmo (2009 s / p)

Pasamos del Estado Liberal al Estado de Bienestar y, recién, llegamos al contexto del Estado Socioambiental, o Estado Constitucional Ecológico Democrático de Derechos, en vista del surgimiento de los derechos transindividuales y universales que, a su vez, tienen en la protección del medio ambiente el ejemplo más significativo. Este nuevo mundo del Estado Ecológico requiere una nueva cultura de ética ambiental en la administración pública y en toda la sociedad, así como una nueva mirada o nueva evaluación de los principios constitucionales, en particular, descrita en este artículo, el principio de eficiencia en la gestión de los recursos públicos por la administración pública brasileña.

Silva (2012, p. 67)analiza el tema,elucidando que,la Declaración de Estocolmoexpresa " los diálogos [que] ocurrieran entre países industrializados y no industrializados, teniendo en cuenta aspectos relacionados con la contaminación del aire, el desarrollo económico y la industrialización"

En este nuevo contexto, la eficiencia ambientales de necesaria aplicación por la Administración Publica. Pues ella, según Sisinno et al (2011) "involucra el uso racional de los recursos naturales y la disminución de la generación y desecho de basura, vertidos y emisiones en el aire. Esta disminución traspasa toda la cadena de ofertantes de bienes y servicios".

Hay que poner el tema de la sostenibilidad en las decisiones administrativas diariasdel Estado, y atentar para la eficiencia de las políticas públicasde gestión de patrimonios públicos. Este tema se pone, sin embargo, de poca prioridad tanto para los organismos públicos como para la comunidad que debe atentar y exigir laobservancia de los principios ambientales por la administración pública.

\section{LA EFICACIA COMO PRINCIPIO QUE FUNDAMENTA LA REALIZACIÓN DE LAS POLÍTICAS PÚBLICASSOSTENIBLESEN LA AUTOGESTIÓN DEL ESTADO}

Las políticas públicas son hechas por agentes legislativos y administrativos del Estado. En estetemaes muy común la producción de normascolegislativas entre los poderes legislativo y ejecutivo. Por esto, una vez positivada la norma que autoriza una política pública, deberán los funcionariospúblicos o particulares en ejercicio de función pública aplicar la regla, sin perjuicio de la naturaleza jurídica obligatoria o discrecional de la política pública.

Por supuesto, la función administrativa es fundamental en el proceso de implementación de políticas. Por lo tanto, hay que entender la función administrativa como una actividad político-jurídica del Estado sea vinculada, sea de aplicación discrecional es necesaria para aplicación de los sistemas legales previamente legislado sea por la movilización, por control o por regulación -ex officio-de recursos financieros, recursos humanos, patrimonio y técnicas de gestión - con o sin la participación, por representación, de la sociedad civil y del sector privado - en la concretización de políticas públicas centradas en derechos colectivos, la protección y la garantía de posibilidad de realización delos derechos privados y en el mantenimiento de sus instituciones y agentes, posibilitando la práctica de actos jurídicos-administrativos y la formación de relaciones jurídicas en el estados, entre los estados, administrativo- civiles, administrativo-comercial, y entre el estado y la sociedad favorecidacon sus servicios y productos . 
De hecho, la actividad administrativa es responsable de la movilización eficiente de los cuatro elementos importantes para el desarrollo y la implementación de la gestión política pública del interés público y la auto-administración estatal. Son ellos: a) los recursos financieros; b) los recursos humanos; c) el patrimonio público y d) las técnicas de gestión.

Este paperlimitarasu enfoque a la preparación y ejecución, por la administración pública, de gestión de activos ambientalmente de forma sostenible y eficiente, específicamente en la adquisición y la movilización de los bienes de uso cotidiano.

Empieza con la cuestión de política pública, cuyo análisis es esencial para entender la toma de decisión de comprar de materiales y cómo su intervención es esencial para el cumplimiento de los principios ambientales y, en particular, de la ecoeficiencia.

\subsection{POLÍTICAS PÚBLICAS: LOS CICLOS DE PROCESO E IMPLEMENTACIÓN}

La política pública es "un conjunto de decisiones conectadas, tomadas por un actor o grupo de actores políticos, en relación con la selección de los objetivos y los medios para alcanzarlos dentro de una situación específica en la que el objetivo de este tipo de decisiones sea, en principio, posible de realizarse por estos actores." (Jenkins, 1978, p. 44)

Las políticas públicas de gestión de patrimonios, de adquisición y movilización de los bienes de uso cotidiano por la administración pública,de forma ambientalmente sostenible y eficiente,estánafirmadas tanto por la Constitución como por la ley aplicable a las licitaciones y contratos. Estoresulta, que la realización de esta política debe observar las etapas del ciclo político y administrativo, de acuerdo con la literatura cultivada, como Howlett (2013).

Etapas del ciclo político y administrativo:

\begin{tabular}{|l|l|}
\hline \multicolumn{1}{|c|}{ Resolución de Problemas Aplicada } & $\begin{array}{l}\text { Etapas en el Ciclo Político } \\
\text { Administrativo }\end{array}$ \\
\hline 1. Reconocimiento del problema; & 1. Organización de agenda; \\
\hline 2. solución propuesta; & 2. formulación de políticas; \\
\hline 3. elección de la solución; & 3. toma de decisiones políticas; \\
\hline 4. realización de la solución; & 4. aplicación de la política; \\
\hline 5. comprensión de resultados; & 5. Evaluación de la política. \\
\hline
\end{tabular}

HOWLETT; RAMESCH; PERL, 2013, p. 15

Cada una de las etapas se estructura en varias etapas y procedimientos, y desde el punto de vista de la incidencia de numerosas variantes sociológicas, económicas, políticas, 
jurídicas, científicas e incluso naturales. Sin embargo, y de forma simplificada se puede decir que en cada etapa hay situaciones estándar que la caracteriza.

En la primera etapa, el montaje de la agenda política trabajando los temas polémicos situaciones complejas de interés - que, una vez vistas e identificadas, reciben, por diversas razones, la atención y la valoración de los agentes encargados de elaborar las políticas públicas, que se repercute, en consecuencia, en sus acciones.

Kingdom (1995) describe la agenda como una lista de preguntas o problemas que reciben verdadera atención por parte de los funcionarios del gobierno, estos o son percibidas por ellos mismos o les presentan por parte de terceros. Por lo tanto, el foco de la agenda política es más pequeño que el universo de temas y problemas reales que afectan la sociedad.

Con la entrada de un tema en la agenda pública y el desarrollo de conciencia de que debe haber algunaposición estratégica e incluso una deliberación, se inicia el proceso de formulación de la política pública, asentado en la posibilidad de crear y evaluar alternativas para proponer soluciones al problema. En esta segunda etapa, por lo tanto, el objetivo es hacer una especificación de posibles opciones de política que podrían utilizarse para abordar las cuestiones y problemas planteados en la agenda (HOWLETT; RAMESCH, PERL, 2013).

La tercera etapa es la toma de decisión política; es decir, se refiere a la dimensión deliberativa del organismo político. Por lo tanto, en esta etapa hay un conjunto de cuestiones por reflexionar, tales como: a) la competencia de decidir; b) el proceso de toma de decisiones; c) los efectos de la deliberación. Esta es laetapa abierta a la decisión sobre la cuestión en la agenda política y las posibles opciones que se presentan al tratar de ella (HOWLETT; RAMESCH, Perl, 2013). Por lo tanto, en esta etapa se debe decidir sobre las cuestiones o problemas que se presentan y elegir sus posibles soluciones alternativas, y para poner en práctica, o no, alguna acción (BREWER; DeLEON, 1983)

En la cuarta etapa - la aplicación de las políticas públicas - es que se debe suscitar la sinergia entre los actores, recursos y procesos necesarios para llevar a cabo la acción. Esta tiene por objetivo lograr la solución que fue decidida por el órgano político. Esta es la etapa más larga y en el que la eficiencia de la gestión y la optimización de los resultados deben ser perseguido constantemente. En consecuencia,

\begin{abstract}
Después de un problema público pudo entrar en la agenda política después de que se formularon varias opciones para solucionarlo y después de que el gobierno estableció los objetivos políticos y se decidió por un curso de acción, aunque depende de él para poner en práctica la decisión. El esfuerzo, los conocimientos y los recursos utilizados en la conversión decisiones políticas en acciones incluyen la etapa de implementación del ciclo politico. Mismo que la mayoría de las decisiones políticas identifica los medios para alcanzar sus objetivos, las opciones posteriores tienen que lograr resultados. Para que una política se realice de verdad, es necesario asignar fondos, designar a las personas y desarrollar reglas sobre cómo proceder. (HOWLETT; RAMESCH, Perl, 2013, p 179).
\end{abstract}

Como corolario del proceso, la quinta etapa es la evaluación de las políticas públicas, no sólo los resultados y los procesos que se llevan a cabo, sino también el aprendizaje que se proporcionó. En este contexto, la quinta etapa es muy importante para evaluar la eficacia y la continuidad, o no, de una determinada medida de política que ha sido adoptada, que permitirátambién su revisión o adaptación para adecuarse al fin perseguido (HOWLETT; RAMESCH, Perl, 2013). Además permite la comprensión de la necesidad de optimizar o detener la aplicación de la política pública analizada y en un Estado democrático de Derecho bien estructurado, puede permitir incluso que los ciudadanos participen en esta composición y la modificación de forma real. 


\subsection{LAS POLÍTICAS PÚBLICAS Y EL PRINCIPIO DE LA EFICIENCIA}

Como decisión de repercusión colectiva, la política pública, en todos sus ciclos, debe estar basada en una adecuada planificación de las necesidades y los intereses reales de la sociedad o fundada en la auto-administración del estado, con el fin de traer respuestas optimizadas a las demandas y problemas.

Para que esto ocurra, la política pública debe ser hecha e efectuada con el apoyo en la recopilación y disponibilidad deinformaciones fiables sobre temas que pueden tener sinergiay relevante interéspúblico, como la sostenibilidad ambiental.

Una vez recogidos y presentada la información, la eficiencia en la política pública requiere una planificación de cómo solucionar el problema, objeto de la decisión. Planear significa tener un análisis real del problema que hay que afrontar, y sobre él, tejer un pronóstico, con el fin intentar solucionar de la mejor manera y lo más pronto posible; es decir, elegir una solución que sea potencialmente "excelente", tanto en su elaboración como en su ejecución.

La eficacia en la política pública requiere continuidad en la gestión que conducen a la decisión política, buscando todaslas cuestiones técnicas y financieras para garantizar su viabilidad y se les ocurran las correcciones necesarias. Por lo tanto, el estado necesita profesionalizar sus agentes y debe requerir profesionalización de los agentes privados que actúen en la administración de cualquier forma. Por otra parte, la responsabilidad fiscal de una política eficaz no puede ser meramente formal, bajo pena de convertirse en frágiles e incluso irrealizables, todas las decisiones. En este sentido, la burocratización debe ser la mínima necesaria para la buena gestión, a fin de no poner en peligro la viabilidad del interés público o de auto-administración del Estado, debe intervenir solamente para permitir su seguimiento y su aplicación efectivas. Al mismo tiempo, las actividades de control interno y externo de la gestión deben ser educativas, y sólo punitivo de forma complementaria.

Por último, la eficiencia en la política pública debe permitir el flujo de ideas, autoevaluaciones y las buenas prácticas, para garantizar una sinergia entre los funcionarios públicos y privados para decidir responsablemente y poner en práctica sus decisiones. Y, por supuesto, en una democracia, los medios de acceso a la información y posibilidades reales de participar en la sociedad - en sus diferentes segmentos y la comprensiones del mundo - deben ser objeto de una mejora constante, especialmente a través de las nuevas tecnologías informatizadas.

Por lo tanto, el Estado debe prestar atención a las dimensiones de la gestión eficiente, que incluye la eficiencia real, eficacia y efectividad.Furtado (2007), adecuadamente, presenta la eficiencia desde el punto de vista de la economía (relación entre costos / beneficios), en correlación con la eficacia de la acción política (evaluación de los beneficios para la sociedad) y su efectividad (si realmente se han alcanzado los objetivos).

En cuanto para Modesto (2015), el contenido del principio de eficiencia es multidimensional, o sea incluye muchos otros elementos, no sólo la eficiencia económica (costo / beneficio). En la comprensión del autor,

La imposición de un desempeño eficiente desde el punto de vista legal, se refiere a dos dimensiones de la actividad administrativa inextricablemente ligado:

a. el tamaño de la racionalidad y optimización en la utilización de medios;

b. el tamaño de efectividad de los resultados de la actividad administrativa pública.

Revista de Direito Brasileira | São Paulo, SP | v. 13 | n. 6 | p. 19 - 38 | jan./abr. 2016 
No se trata sólo de uno o otro requisito, pero los dos combinados. Eficiencia, jurídicamente, no es sólo un uso razonable o correcto de los recursos y medios disponibles en función de los propósitos esperados, como creen los economistas y administradores. La eficiencia para los administradores, es un sencillo problema de optimización dos medios; para el jurista, se refiere tanto a la optimización de los recursos y la calidad del actuar buscando los fines. Se recuerda que el administrador público está obligado a actuartenendo como parámetro el mejor resultado, y referenciado en el principio de proporcionalidad.

A su vez, Batista Júnior (2012, p. 99) afirma que

[...] Para la promoción del bien común, no que se refiere la actuación dela AP [Administración Pública], tanto los medios como los resultados tiene mucha importancia. El PE [principio de eficiencia] por lo tanto es un principio biopotenciales, pues su acción vista de forma jurídica se agobia tanto con a la acción instrumentalcomo con el resultado que se obtiene. El principio requiere tanto el uso máximo de potencialidades existentes, es decir, los recursos escasos que la comunidad tiene, como resultado optimizado cuantitativa y cualitativamente, con respecto a la satisfacción de las necesidades colectivas.

Y lo que sería, en síntesis, la eficiencia de la política pública?

Como una decisión repercusión pública, la actividad política debe basarse en una adecuada planificación de las necesidades y en los intereses reales de la sociedad y de la autogestión delestado, a fin de dar respuestas optimizadas a las demandas de la comunidad y de la administración.

Para que esto ocurra, la política pública debe ser implementado en todas sus etapas, con el respaldo en la recopilación y disponibilidad de información fiable sobre los temas que pueden ser relevantes y que influyen en la sinergia pública, tales como la seguridad (preventiva, represiva y de investigación), el acceso a la educación (fundamental, científica y tecnológica), el acceso a salud (tratamiento y prevención de enfermedades), el acceso a la infraestructura (carreteras, sanitaria y energética), el acceso al mercado de trabajo, el acceso a la actividad empresarial pública y privada, a la sostenibilidad ambiental, entre otros.

Una vez recogida y puesta a disposición la información fiable (aquella que abarca con veracidad y con detalles toda la realidad de su campo de investigación), la eficiencia en la política pública requiere una planificación en relación al objeto que será decidido y posibles medidas de solución. La planificaciónes, entonces, la realización de un diagnóstico real del problema que hay que afrontar, y el desarrollo de pronóstico factible con el fin de resolver de la mejor manera posible y en el menor tiempo; es decir, para buscar una solución que es potencialmente optima, tanto en su preparación como en su aplicación.

Entonces, la eficacia de la política pública requiere continuidad en la gestión de objetos de decisión política, teniendo en cuenta los puntos técnico y financiero para su viabilidad. Por lo tanto, el Estado necesita profesionalizar sus agentes y debe requerir profesionalización de los agentes privado que actúen con él. Por otra parte, la responsabilidad fiscal de una política pública eficaz no puede ser meramente formal, bajo pena de convertirse en inútiles e incluso irrealizables todas las decisiones. En este sentido, la burocratización de los procedimientos deben ser mínimamente necesaria para una buena gestión, a fin de no poner en peligro la viabilidad del interés público, pero sólo controlar garantizando eficacia. Por el contrario, las actividades de control interno y externo de la gestión deben ser principalmente educativa, y sólo punitivo de forma complementaria.

Por último, la eficiencia de la política pública debe permitir el flujo de ideas, garantizando sinergia entre los funcionarios públicos y privados para decidir políticamente y poner en práctica sus decisiones. Y, por supuesto, en una democracia, los medios de acceso a 
la información y posibilidades reales de participación de la sociedad- en sus diferentes segmentos y la comprensión del mundo-que debe ser objeto de una mejora constante, especialmente a través de las nuevas tecnologías informatizadas.

\section{PRINCIPIOS AMBIENTALES Y LA CONSTRUCCIÓN DELPRINCIPIO DE LA EFICIENCIA EN LA ADMINISTRACIÓN PÚBLICA}

La función del Estado en la protección del medio ambiente se define desde del surgimiento del Derecho Ambiental. Para garantizar el cumplimiento de esta función, el Estado tuvo que desarrollar nuevas técnicas cada vez más se modernizar para proteger el medio ambiente, y creando nuevas formas de prestación de servicios públicos, de imponer normas de comportamiento que efectiven la garantía de protección y preservación del medio ambiente y también desarrollar un sistema de estímulos e incentivos a la acción privada que corroboren con los mismos fines. (MOREIRA NETO, 1977). En el Estado de Derecho a actuación del poder estatal se pasa en los límites de la ley que los pre-establece, por esto el Estado debe necesariamente construir un sistema legal que se ocupa de estas nuevas formas de acción del Estado, fundada en principios ambientales establecidos los tratados internacionales reconocidos por él.

El derecho ambiental surge en los años 70, teniendo como marco inicial la Conferencia de Estocolmo de 1972. Como una ciencia autónoma, el derecho ambiental tiene principios solamente suyos que surgen en la Conferencia de Estocolmo de 1972 y se expandieron con la Declaración de Río sobre el Medio Ambiente y el desarrollo de 1992 (FIORILLO, 2009). Para estudiar la inclusión de eco-eficiencia en la administración pública y su importancia en la licitación sostenible, es necesario analizar los principios generales de la legislación ambiental que guían estos procesos.

El principio primero es de la Protección del Medio Ambiente ecológicamente equilibrado como derecho fundamental que, tiene el derecho al medio ambiente como el valor supremo de la sociedad, como extensión del derecho fundamental a la vida, como derecho de tercera generación, o difusa, traída por el Estado Democrático de Derecho (MILARÉ, 2009). Dadas las diferencias entre las sociedades y regiones, el cumplimiento de este principio conduce al segundo principio de acceso equitativo al Medio Ambiente. Por este principio, debe ser asegurado que todostienen derecho a una igualdad material al medio ambiente, tanto para las generaciones presentes como las generaciones futuras.

Otro principio importante es lo de quien contamina, paga. De acuerdo con este, si un agente degrada un bien ambiental, debe realizar la compensación necesaria, para garantizar la protección del derecho de acceso de toda sociedad, que se lesionó con la actitud de aquel. Este principio busca sancionar el acto perjudicial y no dar derecho del delincuente a contaminar como informaMilaré (2009). Este es el principio detrás de la internalización de manera más inmediata de los costes sociales del daño ambiental por el agente que lo causa.

Otro principio esencial es del desarrollo sostenible, es esencial para entender la forma actual de la legislación ambiental y su papel en las licitaciones sostenibles. Surge en los años 80 , como un concepto creado por el Informe Brundtland, y establecido internacionalmente por la ECO / 92. El desarrollo sostenible informa que se debe igualar armónicamente el ciclo de la explotación de los recursos naturales, esenciales para la producción económica, con el ciclo de la biodisponibilidad de estos, lo que resulta en un aumento en el bienestar social, la mejora de las condiciones de vida de esta generación y las futuras (DERANI, 2008). Crea una armonía entre el desarrollo y el medio ambiente, siempre en una perspectiva histórica y 
cultural, garantizando la búsqueda de soluciones adecuadas para cada empresa, y con su participación, para satisfacer sus necesidades materiales, inmateriales y calidad de vida sin dañar el patrimonio ecológico y cultural de la sociedad que en verdad se transforma en forma de también garantizar el desarrollo económico, pero este se dará por medio de los instrumentos más adecuados para minimizar al máximo el coste ambiental de la degradación. Y no se debe confundir con la sostenibilidad que está interesada en el mantenimiento de las existencias de los recursos naturales bajo el argumento de que estas características son esenciales para la continuidad de la actividad económica.

Otros principios clave son la consideración de la variable ambiental en las decisiones públicas, que considera esencial que todas las decisiones adoptadas por los organismos gubernamentalesdeben considerar el impacto y el daño al medio ambiente con el fin de garantizar los principios anteriores.

Por último, pero no menos importante el principio de la naturaleza pública de Protección del Medio Ambiente, que amparalos otros principios, definiendo una función estatal que cubra todas las cuestiones ambientales en todo el sistema productivo de este.

Dicho esto, se tiene que los recursos ambientales, mismo que pertenezcan a particulares, no están disponibles para uso como se quiera, ya que éstos son inseparables del bien común: un medio ambiente ecológicamente equilibrado. Las actividades entonces deben ser reguladas por el Estado para proteger este bien común, sino que también deben cumplir con este precepto. El Estado debe dar el ejemploen sus acciones eincentivar los otros elementos sociales a acciones que conduzcan a impactos positivos y que garanticen la preservación y protección del medio ambiente, ya que este una vez devastado, nunca vuelve a su estado anterior.

En este sentido, la naturaleza jurídica del medio ambiente escomo derecho de tercera generación en la definición de Bobbio (1992), que lo distingue de otros tipos de recursos. En primer lugar porque rompe la división clásica entre la propiedad pública y privada, ya que este bien no está individualmente disponible a nadie, pues es un interés transindividual y también por ser los titulares del medio ambiente indeterminados. En segundo lugar, como pro la naturaleza el medio ambiente es indivisible, como se expone Milaré (2009), pues la calidad del equilibrio ecológico no puede ser individualizada, pasando por el interés de todos. De ahí la necesidad de fomentar una reducción en el uso del medio ambiente, fomentando la producción ambientalmente correcta. Lo que también demuestra la importancia que tiene el gobierno cuando realiza compra de productos que cumplan con los requisitos ambientales y se agobie con los efectos sobre la sociedad en su conjunto, de sus productos.

En este contexto, el derecho y su protección están presentes en toda la cadena de las actividades humanas y pueden ser vistos desde la explotación de recursos naturales que, tras el debido proceso, hasta la aparición de posibles daños en el medio ambiente y para la sociedad misma.

Es decir, el impacto ambiental puede ser positivo o negativo, ya que el efecto que resulta puede ser directo o indirecto, reversible o permanente. De acuerdo con la Resolución CONAMA No 01,1986 :

Artículo 1 - A los efectos de esta Resolución, considerase impacto ambiental cualquier cambio de las propiedades físicas, químicas y biológicas en el medio ambiente, causadas por cualquier forma de materia o energía resultantes de las actividades humanas que afectan directa o indirectamente:

I - la salud, la seguridad y el bienestar de la población;

II - las actividades sociales y económicas; 


\author{
III - la biota; \\ IV - las condiciones estéticas y sanitarias del medio ambiente; \\ V - la calidad de los recursos ambientales. (Medauar, 2014, p.705)
}

Vale la pena mencionar una vez más que el impacto ambiental no es sinónimo de daños al medio ambiente. El primero puede traer beneficios o perjuicios a la esfera en la que se produce. El segundo es necesariamente el daño causado al medio ambiente, entendido en su sentido más amplio, llegando a todos los elementos protegidos por la legislación ambiental y sus instrumentos, que va más allá de lo simples desequilibrio ecológico, porque como dicho, alcanza una colectividad indeterminada de personas. Por lo tanto, ya no es solamenteoponerse el daño causado a los individuos como tales, pero el daño que, al medio ambiente se hace también a toda la comunidad, porque perjudica los intereses difusos. Pero también debe buscarse que las actividades traen consigo impactos más positivos que negativos para el medio ambiente.

Por último, es necesario decir que más allá del concepto de impacto ambiental, la Resolución CONAMA No 01 de 1986 trajo consigo,en el art. $2^{\circ}$, una lista de ejemplos de actividades que impactan en el medio ambiente. Pero esta lista de actividades que se presentan no es exhaustiva ya que los impactos ambientales no son lineales, no se estancan en el tiempo.

Está claro que el derecho al medio ambiente, entendido aquí como fundamental para garantizar el derecho a la vida, está por encima de los beneficios económicos que la compra de productos por "precio más bajo" ofrece a corto plazo. Uno no debe, sin embargo, utilizar el derecho fundamental a un ambiente ecológicamente equilibrado como delimitador pura y simple del desarrollo económico, bajo pena de violar el principio de desarrollo sostenible como explicitado. El uso eficaz de los instrumentos para la protección del medio ambiente está estrechamente ligado a la capacidad del Gobierno para llevar a cabo la situación concreta de garantizar la sociedad al mismo tiempo la protección ambiental y el desarrollo sostenible.

Es necesario que el tratamiento económico de las cuestiones ambientales deba tomarse en serio y ampliamente, ya que sin ellas el panorama económico se presenta de manera miope sin el debido cuidado del medio ambiente, lo que en verdad se ve hoy diuturnamente. Esta distancia entre ambos es la crisis ambiental, pues los procesos económicos están sujetos a una lógica de mercado que conduce a la degradación, ya que no se contabilizan las externalidades negativas ambientales. Es necesario, una reforma en esta visión de lógica de mercado, pues, las externalidades negativas sociales y ambientales se extienden cada vez más debido a la irracionalidad (o racionalidad excesiva) del capital, lo que generará, además de la actual crisis ambiental, la insostenibilidad del propio mercado. Los organismos públicos a través de políticas públicas deben fomentar un proceso económico que cumple con los principios del desarrollo sostenible, ya que actualmente se define en el artículo 3 de la Ley Federal de licitación pública.

La Protección ambiental es de importancia fundamental tanto intergeneracional como inter-social. Cuando se produce el daño y esto no es pagado por los que causaron esto genera una externalidad en el sistema económico, es decir, " costos que afectan a terceros, sin la debida compensación" (MOTTA, 1997, p. 3). Por lo tanto, las externalidades ambientales tienen que ser internalizado en la planificación de las actividades económicas, tales como los costes medioambientales, porque sin esto, genera la apropiación privada del capital natural, creando una masa de excluidos, tanto en el presente como en las generaciones futuras. Por lo tanto, "a pesar de la utilización de recursos ambientales no tener su precio de mercado reconocido, su valor económico existe en la medida en que su uso altera el nivel de producción y consumo (bienestar) de la sociedad" (MOTTA, 1997, p. 3). Más allá de la cuestión económica, hay el problema de las repercusiones culturales, estéticas, en valores 
biológicos, etc. de los bienes ambientales ni todos estos elementos son pasivos de monetización, ya que ni siempre se asocian a uno uso real y materialmente percibido. Así que cuando los productos son comprados por las agencias públicas sin cumplir con los requisitos ambientales, estos se convierten en co-responsables de los daños causados al medio ambiente por la empresa de quien se lo adquirieron los productos. Esto porque no se ha buscado atentar si estas empresas cumplieron los requisitos necesarios para garantizar la preservación y protección del medio ambiente.

No se puede olvidar que el consumismo es una enfermedad en la sociedad global y necesita ser discutido más profundamente. Debe ser demostrando su impacto real en el medio ambiente y esto debe ser informado y se debe requerir la reducción, pues solamente así el individuo cooperar para la protección del medio ambiente de forma continua e intensa, sin dejar estos a los gobiernos y las empresas solamente.

Así, la administración pública juega un papel esencial en esto, pues más de castigar o simplemente incentivar a través de sus acciones, puede cumplir la protección del medio ambiente y fomentar el desarrollo sostenible y el consumo sostenible.

\subsection{ECOEFICIENCIA}

El principio de la eco-eficiencia o eficiencia ambiental, como se ha explicado, buscar el uso más racional posible de los recursos ambientales con el fin de generar un menor impacto sobre el medio ambiente mediante la reducción de residuos y emisiones en su conjunto, que debe ser aplicado en toda la cadena de suministro. Este principio es esencial en el actual estado, que debe buscar cumplir su obligación ética con el medio ambiente, establecido en el artículo 225 de la Constitución Brasileña y en lasDeclaraciones de Estocolmo (1972) y Río (1992), ambos firmados por Brasil. Por lo tanto, debe intentar, para cumplir con este principio, un consumo más bajo con un uso más eficiente, seleccionando como prestadoras,empresas que ofrecen servicios y materiales que cumplan con los principios del desarrollo sostenible y la sostenibilidad.

En la crisis actual de valores que vive Brasil, donde el individuo prevalece sobre el colectivo, la aplicación de la ecoeficienciapermiteservidor público desarrollar una administración pública eficaz y capaz de satisfacer los intereses colectivos, porque posibilitaría reducir el desperdicio, la ineficiencia y reducir el desperdicio de uso de los bienescomunes de la sociedad. Además influí positivamente en las empresas que compiten en las licitaciones con el fin de hacerlos más respetuosos del medio ambiente, e influyen en una aplicación real de los requisitos ambientales de los competidores, que luego van a preocuparse no sólo con el desarrollo económico, sino también con la protección y menor uso del medio ambiente y el desarrollo social de Brasil, en el cumplimiento del principios del desarrollo sostenible.

Esta disposición tiene por objeto cumplir con los criterios del artículo 37 de la Constitución Brasileña, y especialmente comprender que la Constitución es un todo que se interrelaciona y que los artículos deben ser leídos como si formasen un todo y deben aplicarse en conjunto, de acuerdo con la perspectiva Dworkiana (Dworkin, 1999), donde se predice que el derecho debe ser aplicado como un solo bloque de normas.

La compartimentación del derecho en gran parte también obstaculiza la aplicación de las normas ambientales, pues el Derecho Ambientalno debería ser y no se colgó a cualquier área del derecho, sino impregna todos ellos, incluido el derecho administrativo. 
Así que, para aplicar el artículo 37 de la Constitución, este debe ser considerado en relación con el artículo 170, inc. VI y el artículo 225, formando un todo en la Unión para llevar a cabo la compra en los procesos de licitación debe analizar no sólo los requisitos administrativos, sino también los derechos al medio ambiente y los derechos humanos. Según Valente (2011, p. 7) "debe tener en cuenta, debido a su función de defender y preservar el medio ambiente, la elección de los productos, servicios y bienes que cumplen los criterios de sostenibilidad ambiental, sin embargo, sin imponer restricciones que comprometen la igualdad de trato en el proceso competitivo ".

Ecoeficiencia segundo Holmo (2009, s/p) "fomenta el consumo más bajo, elincremento de la producción, incluso ofreciendo la posibilidad de nuevos usos para los mismos productos incluidos en el proceso, y estimular la competitividad entre las empresas". Las empresas públicas serían ejemplos a seguir y cumplirían el interés común de la protección ambiental previsto en la Constitución Federal.

Las ganancias en la aplicación de la ecoeficiencia no son sólo de carácter económico y ambiental, sino también social. Con el despertar la conciencia de los trabajadores de los riesgos para la sostenibilidad de las generaciones futuras causadas por el impacto negativo de sus acciones sobre el medio ambiente, genera un cambio de visión, lo que resulta en un cambio de actitud que termina siendo difundido más allá del escritorio ( SISINNO, RIZZO, SANTOS, 2011, p.25)

La Ecoeficiencia aparece por primera vez en el contexto nacional en el A3P "que constituye una visión integrada de la gestión pública, en donde se logra la reducción de la degradación del medio ambiente mediante la reducción de residuos y del impacto ambiental y la optimización de procesos, centrándose en la prevención. "(HOLMO,2009, s/p) Hoy estoencuentra apoyo legal en el artículo 3 de la Ley $n^{\circ}$ 8666, de 1993 y el Decreto $n^{\circ} 7746$ de 2012, tema discutido en la sección abajo.

\section{LA ADMINISTRACIÓN PÚBLICA FEDERAL Y EL PRINCIPIO DE EFICIENCIA EN LAS LICITACIONES EN BRASIL}

La Agenda 21 ya especificaba el principio de la consideración de la variable ambiental en las decisiones públicas. Comprar productos es acto esencial para mantener los órganos en funcionamiento y la elección del proveedor dentro de los criterios legales es una importante decisión pública y que genera altos costos en mediano plazo, si el problema ambiental es ignorado. Hay que resonar que la administración pública es un importante comprador y que sus acciones o requisitos para comprapueden condicionar el mercado productor negativa o positivamente en relación con el uso del medio ambiente.

Pensando en esta cuestión, el Ministerio de Medio Ambiente propuso en 1999, la Agenda Ambiental de la Administración Pública (A3P) que tiene por objeto impulsar el gobierno federal a incluir criterios ambientales en licitaciones públicas.

Aunque no sea de naturaleza dictadora, a A3P ha traído a la reflexión la idea de la gestión ambiental en el poder público federal, y estimuladas prácticas ambientales más racionales en todas las áreas y procedimientos administrativos. Para comprender el impacto que la cuestión del medio ambiente es importante recordar que, según Valente (2011), se estima el $15 \%$ de PIB viene de la contratación pública. Es un número muy significativo, y si la administración publica actuar o exigir la gestión ambiental aplicada junto con la producción sostenible en las empresas que participan en la licitación pública, este acto puede tener un 
enorme impacto positivo en la protección del medio ambiente, proporcionando una ampliación de las externalidades positivas.

Buscando este efecto, el misterio de planificación ygestión crea la Instrucción Normativa $\mathrm{N}^{\mathrm{o}} 1$ de 19 de enero de 2010 , que propone criterios de sostenibilidad ambiental en la adquisición de bienes, contratación de servicios o de obras para la Administración Pública Federal directa y autárquica, como forma de establecer un marco regulador para adoptar criterios ecológicos y sostenibles para la contratación pública y el establecimiento de las compras públicas sostenibles. Busca también estimular la competición entre las industrias para uno mejor desempeño ambiental de sus productos en todas las etapas y, al mismo tiempo ganando precios bajos y una ofertamejor. Así, o proceso y su reglamento realizan la ecoeficiencia, que, también debe ser buscada como criterio en las inversiones, compras y adquisiciones del gobierno federal.

La Guía de Adquisiciones Sosteniblepara la Administración Pública Federal (2011), dividió en tres tipos principales los productos que se consumen por la Administración Pública Federal

Inicialmente hay los insumos, que se denominan materiales de oficina tales como papel, productos de limpieza, equipaos técnicos y tecnología de la información, los muebles. En general, son bienes de consumo durables y materiales.

El segundo tipo de producto son los servicios como el mantenimiento, la limpieza, el apoyo técnico para los equipos, etc.

Y, por último, hay las obras, por ejemplo, proyectos de ingeniería civil, como carreteras, edificios públicos, puentes, etc.

En este trabajo nuestra atención se dirige a la compra de insumos. Especialmente, los más consumidos y comunes pero lo que menos se ven, a pesar de que se necesitan en todasorganizaciones. Lápiz, bolígrafos, gomas de borrar, grapadoras, papel, etc que son vistos como pequeños objetos de uso diario, que no interfieran con fuerza en el presupuesto público o como dañosos al medio ambiente, como se pasa con las obras y grandes servicios.

Estos pequeños objetos de medio y largo plazo de uso, tienen un impacto significativo, y por ser parte del día a día normal, terminan convirtiéndose en invisibles y por lo tanto ignorados cuando se piensa en el evento de compras sostenibles. No se observa cómo se los hicieran, si es realmente eficiente, producidos dentro con atención a las cuestiones ambientales y si las fábricas cumplen con su papel del desarrollo de la sociedad brasileña. Esta no preocupación que agrava el problema del medio ambiente, y el cumplimiento de un desarrollo sostenible y la prestación de ecoeficiencia.

Teniendo en cuenta esta perspectiva,la Presidencia de La Republica Brasileña propuso la Medida Provisional $\mathrm{n}^{\mathbf{0}} 495$ de 2010, cambiando la redacción del artículo 3 de la Ley $\mathrm{n}^{\circ}$ 8666 del año 1993 con el fin de introducir el desarrollo nacional como precepto en la ley. El Congreso no tuvo dudas y ver la perspectiva de la aplicación de la Constitución en su unicidad añadió el término "sostenible" cuando se transformó la Medida Provisional no 495 en la Ley $n^{\circ} 12.349$ de 2010.

O Congreso innovó en la interpretación al modificar la Ley 8666/93 realizando la inserción en su redacción que la contratación pública debe ser sostenible:

Art. $3 \mathrm{La}$ licitación está destinada a garantizar el cumplimiento del principio constitucional de la isonomía, la selección de la propuesta más ventajosa para la gestión y promoción del desarrollo nacional sostenible y será procesado y juzgado en estricta conformidad con los principios básicos de legalidad, impersonalidad, 
moralidad, igualdad, publicidad, probidad administrativa, do enlace con el anuncio de la oferta, el juicio objetivo y todo que les están relacionados. (Brasil, 1993)

Esto aseguró la obligatoriedad de que las licitaciones tengan en cuenta el principio del desarrollo sostenible y la sostenibilidad no sólo en las licitaciones públicas federales, pero en todas que lo quisieren.

La administración, entonces, más que moralizar la contratación pública,asume su papel de proteger el medio ambiente y de convertir esta nueva realidad social mirando garantizar la sostenibilidad y la conciencia ambiental. Esto es, además, de seguir el "LIMPE" definido por Meirelles, Kamimura, y Oliveira (2012, p.3) como "la legalidad, impersonalidad, moralidad, publicidad y eficiencia, principios previstos para todos los actos realizados por el gobierno [...]" pasa, entonces, a guiarse también por los criterios que conducen a la reducción de externalidades negativas ambientales y daños al medio ambiente, y a adoptar criterios de sostenibilidad para las compras licitadas.

Reglamentando la Ley $n^{\circ} 8666$, de 1993, modificada por la Ley ${ }^{\circ} 12.349$, de 2010, se emitió el Decreto n ${ }^{\circ} 7746$ de 2012, que estableció directrices para la realización de las licitaciones sostenibles y parámetros de sostenibilidad debe ser respetados.

El Decreto $\mathrm{n}^{\circ} 7746$ de 2012, pone en práctica lo que ya estaba establecido en el artículo 3 de la Ley de Licitaciones; en el artículo 2, el Decreto establece que "La administración pública federal directa, agencias y fundaciones autónomas y empresas públicas pueden adquirir bienes y contratar servicios y obras teniendo en cuenta los criterios y las prácticas sostenibles objetivamente definidos en el instrumento de convocación, según lo previsto en el presente Decreto. (Brasil, 2012)

El art. 4 presenta directrices que implementan claramente el principio de desarrollo sostenible en los certámenes de la Administración Pública clara e inequívoca, siempre que los requisitos sean claros y se presentan en el texto de convocación, de forma que no haya diferenciación o restricción que infrinja los principios de Derecho constitucional o administrativo. Entre estos requisitos pueden ser requeridos que los productos sean hechos de material reciclado, no tóxico o biodegradables, y otros criterios de sostenibilidad.

Novedad establecida en el Decreto, que pretende dar cumplimiento a la norma, es la institución, en el art. 9, de la Comisión Interministerial de sostenibilidad en la Administración Pública - CISAP,

Art. 9 [...] de naturaleza consultiva y permanente, vinculado al Departamento de Logística y Tecnología de la Información, con el fin de proponer la aplicación de criterios, prácticas y acciones logísticas sostenibles dentro de la administración pública federal directa, autárquica, de fundaciones y empresas públicas.

\section{Art. 10. El CISAP consistirá en:}

I - dos representantes del Ministerio de Planificación, Presupuesto y Gestión, de la siguiente manera:

a) un representante de la Secretaría de Logística y Tecnología de la Información, que será el presidente; y

b) un representante de la Secretaría Federal de Presupuesto;

II - un representante del Ministerio de Medio Ambiente, que ejercerá la vicepresidencia;

III - un representante de la Casa Civil de la Presidencia;

IV - un representante del Ministerio de Minas y Energía;

V - un representante del Ministerio de Desarrollo, Industria y Comercio Exterior; 


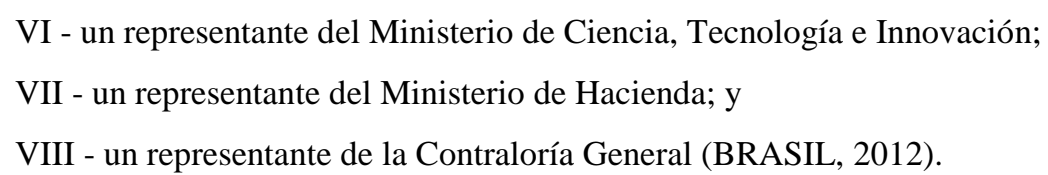

Esta Comisión tiene la tarea de organizar el proceso de adopción en perspectiva nacional del principio del desarrollo sostenible para toda la administración pública federal directa, los organismos autónomos y fundaciones y empresas públicas de forma que estos desarrollen e implementen planes de gestión de logística sostenible.

La ley innova en el proceso de licitación, pero todavía sufre del mal de lenta implementación en forma amplia. Pero es un gran paso hacia la protección del medio ambiente por las agencias estatales de Brasil.

\section{CONCLUSIÓN}

La consideración de la sostenibilidad ambiental como parámetro de eficiencia en el desarrollo de políticas públicas para la adquisición materiales consumibles en la práctica diaria de la administración pública hoy en día es fundamental para el cumplimiento de la responsabilidad del Estado en proporcionar la aplicación de los principios del derecho ambiental y también torna eficazel principio de la eficiencia en la toma de decisiones e implementación de políticas. Esto, como presentado, se ha convertido en un parámetro importante para medir el diseño y la implementación de los intereses públicos, e incluso de los intereses privados, que pueden ser considerados relevantes para la viabilidad de los servicios y las ofertas de bienes relacionados con la mejora de la vida pública en sociedades complejas. Aunque, todavía, no se ha alcanzado la convenida importancia en la elaboración de políticas públicas en relación a las actividades habituales de auto-gestión de los organismos estatales. Esto se debe a poca eficiencia que las instituciones y agencias estatales en el manejo de los recursos y bienes del Estado, especialmente en los aspectos que aparentemente son de poco valor o importancia.

La protección del medio ambiente es hoy uno de los puntos más importantes de preocupación internacional y nacional, desde la Conferencia de Estocolmo, 1972, que trajo las responsabilidades del Estado con la protección del medio ambiente. La Conferencia de Río/92 a posterioriincluyó los principios del desarrollo sostenible y la consideración de la variable ambiental en las decisiones públicas propició la realización de la ecoeficiencia, buscando así la sostenibilidad del medio ambiente en la autogestión cotidiana del Estado y la eficiencia de las políticas públicas de bienes públicos.

Como indicado, aunque existe un incentivo legal, laeco-eficiencia y la implementación del desarrollo sostenible en las licitaciones públicas en Brasil es poco utilizado, sea por desconocimiento de la norma, sea por falta de penalización por no usarla. Fue comprobado que la política pública de gestión sostenible de activos ambientales y la eficiente adquisición y movilización de uso diario por la administración pública está apoyada tanto por la Constitución como en la ley aplicable a las licitaciones y contratos públicos.

Pero no basta sólo la ley, para que esto suceda, la política pública debe ser diseñada e implementada con el apoyo en la recopilación y garantía de acceso a información fiable sobre cuestiones que pueden ser de relevante interés y sinergia públicos, como es el caso para la sostenibilidad ambiental. 
Por lo tanto, el estado necesita profesionalizar sus agentes y debe requerir profesionalización de los agentes privados que actúen en la administración de cualquier forma. En este sentido, la burocratización debe ser la mínima necesaria para la buena gestión, a fin de no poner en peligro la viabilidad del interés público o de auto-administración del Estado, debe intervenir solamente para permitir su seguimiento y su aplicación efectivos.

Por último, la eficiencia en la política pública debe permitir el flujo de ideas, autoevaluaciones y las buenas prácticas, para garantizar una sinergia entre los funcionarios públicos y privados para decidir responsablemente y poner en práctica sus decisiones.. Por lo tanto, el principio de la consideración de la variable ambiental en las decisiones públicas es esencial, pues es esencial que todas las decisiones adoptadas por los organismos gubernamentales consideren el impacto y el daño al medio ambiente de forma a garantizar los principios presentados.

La eco-eficiencia, como se ha explicado, buscar el uso más racional posible de los recursos ambientales con el fin de generar un menor impacto sobre el medio ambiente mediante la reducción de residuos y emisiones en su conjunto, que debe cumplirse en toda la cadena de suministro. En la crisis actual de valores que vive Brasil, donde el individuo prevalece sobre el colectivo, la aplicación de la eco-eficiencia permiten al profesional pública desarrollar una administración pública eficaz y capaz de satisfacer los intereses colectivos, porque conduciría a la reducir del desperdicio, de la ineficiencia y ayudaría a reducir el desperdicio del bien de uso común del pueblo, que es el medio ambiente.

Por lo tanto, las normas buscan realizar el principio de la ecoeficiencia, que también debe ser buscado como criterio para las inversionesfinancieras, compras y adquisiciones del Gobierno Federal. Y, la administración, más que moralizar la contratación pública, debe asumir su función de proteger el medio ambiente y se convertir a esta nueva realidad social mirando hace adelante con a ejecución de la sostenibilidad y de la conciencia ambiental.

La modificación del artículo 3 de la ley de Licitaciones y el Decreto n 7746 de 2012, como se demostró, innovan en el proceso de licitación, pero todavía sufre de la lenta implementación. Pero ella es un gran paso hacia la protección del medio ambiente por los órganos estatales de Brasil, en cumplimiento a la Agenda 21,los requisitos ambientales y el desarrollo sostenible.

La no realización de estas normas en la realidad, sin embargo, mantiene la licitación pública como un proceso que no cumple con el papel del Estado como gestor quebusca la protección ambientaly, en última instancia genera diversas distorsiones como: la falta de estrategias para mejorar procesos de contratación pública con el fin de hacerlos más flexibles, con reducción de requisitos excesivos que ralentizan el proceso de adquisición; disminuir el problema de que los administradores hagan de los asuntos públicos una extensión de sus hogares, lo que demuestra la necesidad de cambiar la actitud de la sociedad en su conjunto; y también debe ayudar el cumplimiento de las cuestiones ambientales en la administración pública. Para esto, hay que cambiar la cultura sobre el tema, a través de la educación y el desarrollo de la conciencia ambiental. Otro problema es la dificultad de la participación de todo tipo de empresas, siempre se debe dar prioridad a las que cumplen con los principios del desarrollo sostenible y en última instancia, cambiar la cultura de gobierno en relación a la compra de productos observando sólo el precio más bajo en el corto plazo, sin un análisis del ciclo de vida del producto, pues tiene en cuenta sólo el valor final. Con el ciclo de vida aplicado a la contabilidad, se comprueba que el producto más adecuado desde el punto de vista del medio ambiente, en realidad es más económicoen el largo plazo. 
A pesar de este contexto aun desolador, el Decreto $n^{\circ} 7746$ de 2012, trae optimismo de que una nueva cultura de ética ambiental comienza a introducirse en la Administración Pública de Brasil, en pasos lentos, pero es el comienzo de un proceso que puede modificar toda una mentalidad y una realidad económica y empresarial, crenado una nueva forma de protección eficaz del medio ambiente en Brasil.

\section{BIBLIOGRAFIA}

BATISTA JÚNIOR, Onofre Alves. O princípio constitucional da eficiência administrativa. Belo Horizonte: Fórum, 2012.

BOBBIO, Norberto. A Era Dos Direitos $7^{\mathrm{a}}$ reimp. Tradução Carlos Nelson Coutinho. Rio de Janeiro: Campus, 1992.

BRASIL. Lei $n^{\circ}$ 8.666, de 21 de junho de 1993 . Regulamenta o art. 37, inciso XXI, da Constituição Federal, institui normas para licitações e contratos da Administração Pública e dá outras providências. Disponível em <http://www4.planalto.gov.br/legislacao > acessado em $2 / 4 / 2015$.

BRASIL. Decreto $n^{o} 7.746$, de 5 de junho de 2012. Regulamenta o art. 3 o da Lei no 8.666, de 21 de junho de 1993, para estabelecer critérios, práticas e diretrizes para a promoção do desenvolvimento nacional sustentável nas contratações realizadas pela administração pública federal, e institui a Comissão Interministerial de Sustentabilidade na Administração Pública CISAP. Disponível em <http://www4.planalto.gov.br/legislacao > acessado em 2/4/2015.

BRASIL. Guia de Compras Públicas Sustentáveis para Administração Federal. Disponível em: <http://www4.planalto.gov.br/legislacao > acessado em 2/4/2015.

BRASIL. MINISTERIO DO PLANEJAMENTO. Instrução normativa no 1, de 19 de janeiro de 2010. Dispõe sobre os critérios de sustentabilidade ambiental na aquisição de bens, contratação de serviços ou obras pela Administração Pública Federal direta, autárquica e fundacional e dá outras providências. . Disponível em $<$ http://www4.planalto.gov.br/legislacao > acessado em 2/4/2015.

BREWER, Gary D., DeLEON, Peter. The Foundation of Policy Analysis.Monterey, CA: Brooks/Cole, 1983.

COGO, Giselle Alves Da Rocha. A Sustentabilidade Na Administração Pública Federal: Um Desafio Às Organizações. Monografia apresentada na Universidade Tecnológica Federal Do Paraná Programa De Pós-Graduação Em Engenharia De Produção Curso De Especialização Em Gestão Industrial: Conhecimento E Inovação. Ponta Grossa: 2011. Disponível em < http://repositorio.roca.utfpr.edu.br/jspui/bitstream/1/1386/1/PG_CEGI-CI_VII_2011_11.pdf> acessado em 2/4/2015.

DERANI, Cristiane. Direito ambiental econômico. $3^{\text {a }}$ Edição. São Paulo: Saraiva, 2008.

DWORKIN, Ronald. O império do Direito. Tradução Jefferson Luiz Camargo. São Paulo: Martins Fontes, 1999. 
FIORILLO, Celso A. P. Política Nacional do Meio Ambiente. In: Curso de direito ambiental brasileiro. 10ª ed. rev., atual. eampl. São Paulo: Saraiva, 2009. Cap. II, p.10-73.

FURTADO, Lucas Rocha. Curso de direito administrativo. Belo Horizonte: Fórum, 2007.

HOLMO, Karina Augusta Tambara Velho.Para Além da Eficiência Pública: a Ecoeficiência. In: Revista Direito e Paz, 2009. Disponível em <file://C:/Users/Maraluce/Downloads/c55609e9b943df6d1109213c127b2b70\%20(2).pdf > acessado em 2/4/2015.

HOWLETT, Michael, RAMESCH M., PERL, Anthony. Politica pública: seus ciclos e subsistemas; uma abordagem integradora. Rio de Janeiro: Elsevier, 2013.

JENKINS, William I. Policy Analysis: a Polítical and Organizational Perspective. London: Martin Robertson, 1978.

KINGDOM, John W. Agendas, Alternatives and Public Policies.Boston: HarperCollins, 1995.

LAKATOS, Eva Maria; MARCONI, Marina de Andrade. Metodologia do Trabalho Científico: Procedimentos básicos, pesquisa bibliográfica, projeto e relatório, publicações e trabalhos científicos. $7^{\mathrm{a}}$ ed. 5 reimp. São Paulo: Atlas, 2010.

MEDAUAR, Odete (org.). Constituição Federal, Coletânea de Legislação de Direito Ambiental. 13a ed. rev. atual. eampl. São Paulo: Editora Revista dos Tribunais, 2014. RT Mini-códigos.

MEIRELLES, Virgilio Ricardo Coelho. KAMIMURA, Quesia Postigo. OLIVEIRA, Edson Aparecida AraujoQuerido . As Vantagens Da Licitação Sustentável Para Administração PúblicaIn: The 4th InternationalCongressonUniversity-IndustryCooperation - Taubate, SP Brazil - December 5th through 7th, 2012 Disponível em <http://www.unitau.br/unindu/artigos/pdf528.pdf> acessado em 2/4/2015.

MILARÉ, Edis. Direito do ambiente: doutrina, jurisprudência e glossário. $5^{\text {a }}$ ed. rev., atual. eampl. São Paulo: RT, 2009.

MODESTO, Paulo. Notas para um debate sobre o princípio da eficiência: $<$ http://www.planalto.gov.br/ccivil_03/revista/Rev_18/Artigos/art_paulomo.htm>, acessado em 8/3/2015.

MOREIRA NETO, Diogo de Figueiredo. Introdução ao direito ecológico e ao direito urbanístico: instrumentos jurídicos para um futuro melhor. 1977 (nota explicativa, ecologia, o estado e a ecologia; direito ecológico; perspectivas;urbanismo; urbanismo e ecologia;conceito de direito urbanístico.)

MOTTA, Ronaldo Serôada.Manual para valoração econômica de recursos ambientais. IPEA- Coordenação de Estudos do Meio Ambiente e COBIO/MMA,1997. Disponível em: <http://www.em.ufop.br/ceamb/petamb/cariboost_files/manual_20serroa_20motta.p> Acessado em 2/3/2012. 
OLIVEIRA, Márcio Luís de. A constituição juridicamente adequada; transformações do constitucionalismo e atualização princípiológica dos direitos, garantias e deveres fundamentais. Belo Horizonte: Arraes, 2013.

OLIVEIRA, Márcio Luís de. A cultura da ineficiência política das gestões públicasbrasileiras. Editorial. Revista Brasileira de Direito Municipal-RBDM; Belo Horizonte, ano 14, $\mathrm{n}^{\circ}$ 51, p. 1 e segs, jan./mar. 2014.

SILVA, Rodrigo Zouainda. Os desafios do direito ambiental no limiar do século XXI diante da ineficácia do sistema jurídico ambiental brasileiro. Revista Veredas do Direito. Belo Horizonte, vol. 9, n. 18, p. 57-87, jul/dez. 2012.

SISINNO, Cristina Lúcia Silveira. RIZZO, Andréa C. Lima, SANTOS Ronaldo L. Corrêa dos. Ecoeficiência aplicada à redução da geração de resíduos sólidos. Rio de Janeiro: CETEM/MCT, 2011. 29p. (Série Estudos e Documentos, 79)Disponível em < http://www.cetem.gov.br/publicacao/series_sed/sed-79.pdf> acessado em 2/4/2015.

VALENTE, Manoel Adam Lacayo. Marco Legal Das Licitações E. Compras Sustentáveis Na Administração Pública. Brasilia: Câmara dos Deputados, MARÇO/2011. Disponível em $<$ http://www2.camara.leg.br/documentos-e-

pesquisa/publicacoes/estnottec/tema1/2011_1723.pdf> acessado em 2/4/2015. 